Research Paper

\title{
MRPL27 contributes to unfavorable overall survival and disease-free survival from cholangiocarcinoma patients
}

\author{
Liping Zhuang1, Zhiqiang Meng ${ }^{\circledR}{ }^{\bowtie}$, Zongguo Yang ${ }^{2 \bowtie}$ \\ 1. Department of Integrative Oncology, Fudan University Shanghai Cancer Center, Shanghai 200032, China. \\ 2. Department of Integrative Medicine, Shanghai Public Health Clinical Center, Fudan University, Shanghai 201508, China. \\ $\square$ Corresponding authors: Zhiqiang Meng, MD, PhD, Fudan University Shanghai Cancer Center, Shanghai 200032, China. Email: mengzhq@yeah.net; Zongguo \\ Yang, MD, PhD, Shanghai Public Health Clinical Center, Fudan University, Shanghai 201508, China. Email: yangzongguo@shphc.org.cn. \\ (c) The author(s). This is an open access article distributed under the terms of the Creative Commons Attribution License (https://creativecommons.org/licenses/by/4.0/). \\ See http://ivyspring.com/terms for full terms and conditions.
}

Received: 2020.07.17; Accepted: 2020.12.22; Published: 2021.01.01

\begin{abstract}
Objective: This study aimed to investigate the roles of MRPL27 in survival from cholangiocarcinoma patients in The Cancer Genome Atlas (TCGA) database.

Methods: In TCGA-CHOL profile, MRPL27 gene expression and clinical data were obtained. Cox regression models were used to evaluate the potential links between MRPL27 and cholangiocarcinoma survival. Enrichment analysis of MRPL27 was conducted in Metascape and Gene Set Enrichment Analysis (GSEA) databases.

Results: 36 cholangiocarcinoma patients were included in this analysis. MRPL27 mRNA was significantly upregulated in tumor tissues in cholangiocarcinoma patients including intrahepatic, distal and hilar/perihilar cholangiocarcinoma cases (all $p<0.01$ ). Cholangiocarcinoma patients with high MRPL27 had worse overall survival (OS) and disease-free survival (DFS) compared to those with low MRPL27 (all $P<0.05)$. Univariate and multivariate Cox models indicated that MRPL27 should be a risk factor for the OS and DFS in cholangiocarcinoma patients (both $p<0.01$ ). Bioinformatic analysis revealed that MRPL27 mainly involved in the processes of mitochondrial translation elongation, respiratory electron transport, ATP synthesis, and inner mitochondrial membrane organization. No mutations of MRPL27 were screened in cholangiocarcinoma patients.
\end{abstract}

Conclusion: Upregulated in tumors, MRPL27 contributes to unfavorable survival in cholangiocarcinoma patients.

Key words: Cholangiocarcinoma; MRPL27; Ribosome; Overall survival; Disease-free survival

\section{Introduction}

Cholangiocarcinoma was the most frequent malignancy of the biliary system[1]. Compared to hepatocellular carcinoma, the incidence of cholangiocarcinoma was relatively $\operatorname{low}[1,2]$. However, in United States, the incidence of intrahepatic cholangiocarcinoma (ICC) increased with $4 \%$ annually during 1992 to 2000[3] and doubled from 1976 to 2000[2], and these similar trends were also reported worldwide[4, 5]. Even worse, the prognosis of cholangiocarcinoma is devastating because of its silent clinical character, difficulties in diagnosis and limited therapeutic strategies[6]. The median survival in less than 2 years, the average 5-year survival rate of cholangiocarcinoma is approximately $5 \%$ and the mortality of ICC has increased globally[6-8].

Staging systems of cholangiocarcinoma is of great importance for providing information on the prognosis and guidance for therapy. Unfortunately, the majority of the current existed staging systems have proven insufficient for selecting of therapeutic options and lacked prognostic accuracy[6]. Hence, there is an urgent need for reliable prognostic indicators that will enable optimal therapeutic allocation and prognostication. Ribosomes are required for protein production, and are involved in the process of cell proliferation, growth and survival[9]. As member of ribosome pathway, MRPL27 might contribute to the carcinogenesis 
progression in human cancers including cholangiocarcinoma. However, no study has focused on the oncogenic roles of MRPL27 in cholangiocarcinoma.

In this study, we investigated the expression levels of MRPL27 and correlated it with survival in cholangiocarcinoma patients, in the hope that our findings could be helpful for understanding of pathological process and aggressiveness in cholangiocarcinoma, and useful for identifying novel therapeutic target for cholangiocarcinoma precise treatment.

\section{Materials and Methods}

\section{Data resource}

Patients diagnosed with cholangiocarcinoma from The Cancer Genome Atlas (TCGA) database were included in this study. Transcriptome profiling of MRPL27 with Fragments Per Kilobase per Million (FPKM) standardized were obtained from TCGA (https://portal.gdc.cancer.gov/), and clinical data of cholangiocarcinoma (TCGA, PanCancer Atlas) was downloaded from cBioPortal for cancer genomics (http://www.cbioportal.org/)[10, 11]. VLOOKUP index in EXCEL was used to match the gene expression and clinical data. A total of 36 cases were included in this analysis.

\section{Survival analysis}

The survival analysis of MRPL27 for cholangiocarcinoma patients in TCGA-CHOL profile were conducted in Gene Expression Profiling Interactive Analysis web service (GEPIA, http://gepia.cancer-pku.cn/index.html). Outcomes including overall survival (OS) and disease-free survival (DFS) were investigated. Validation analysis of OS and DFS in our matched database was also performed.

\section{Protein-protein interaction (PPI) and enrichment}

PPI of MRPL27 was conducted in Search Tool for Retrieval of Interacting Genes/Proteins (STRING) online service[12]. Interacted genes of MRPL27 were also identified in Search Tool for Interacting Chemicals (STITCH) database[13]. Top 50 similar genes of MRPL27 were identified in GEPIA database[14].

\section{Functional Enrichment of interacted genes of MRPL27}

Interacted genes and similar genes of MRPL27 in STRING, STITCH and GEPIA were included in the enrichment analysis in Gene Set Enrichment Analysis (GSEA) molecular signatures database[15, 16] and
Metascape web service[17]. Top 10 terms of Kyoto Encyclopedia of Genes and Genomes (KEGG) pathway, Gene ontology (GO) biological process and Reactome were described.

\section{Identification of MRPL27 mutations}

Mutations of MRPL27 in cholangiocarcinoma patients were identified in cholangiocarcinoma dataset (TCGA, PanCancer Atlas) from cBioPortal for cancer genomics (http://www.cbioportal.org/)[10, 11]. Mutations including missense, truncating, inframe, fusion and others were all screened.

\section{Statistical analysis}

Differences of variables between the individual groups were analyzed using student $t$ test and MannWhitney $U$ test based on variables types. Factors associated with the survival were assessed by univariate analysis and multivariate analysis Cox regression. Results were reported as hazard ratios (HR) with 95\% confidence intervals (CI). The Kaplan-Meier method with log rank test was used to compare OS and DFS between different groups. Stata software version 16.0 (Stata Corp LLC, Texas, USA) was used. A two-tailed $p<0.05$ were considered significant for all tests.

\section{Results}

\section{MRPL27 expression in cholangiocarcinoma tissues}

As shown in Figure 1, MRPL27 mRNA was significantly upregulated in tumor tissues compared to adjacent normal tissues in cholangiocarcinoma patients $(\mathrm{p}=0.003$, Figure $1 \mathrm{~A})$. According to the tumor locations, MRPL27 mRNA was significantly overexpression in tumor tissues in intrahepatic cholangiocarcinoma, distal cholangiocarcinoma and hilar/perihilar cholangiocarcinoma patients $(\mathrm{p}=$ $0.003, p=0.004$ and $p<0.001$, respectively, Figure 1B-1D). MRPL27 protein staining was identified in the Human Protein Atlas (https://www.proteinatlas .org/). MRPL27 protein was low stained in three normal liver tissues, while medium stained in two of four cholangiocarcinoma tissues (Figure 1E). In addition, we compared the baseline characteristics of cholangiocarcinoma patients between MRPL27 high and low expression groups. As summarized in Table 1 , age, gender, body mass index (BMI), race, family history of cancer, risk factor of cancer, surgical procedure, pathological histology grade, American Joint Committee on Cancer (AJCC) stage, vascular invasion, perineural invasion, fibrosis, new tumor events, alpha-fetoprotein (AFP), total bilirubin, albumin and creatinine levels were equally distributed between the two groups (Table 1). 
(A) Cholangiocarcinoma

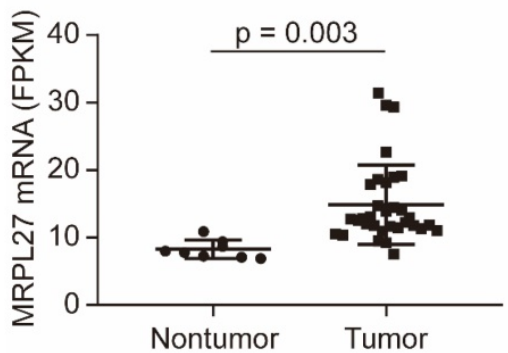

(C) Distal cholangiocarcinoma

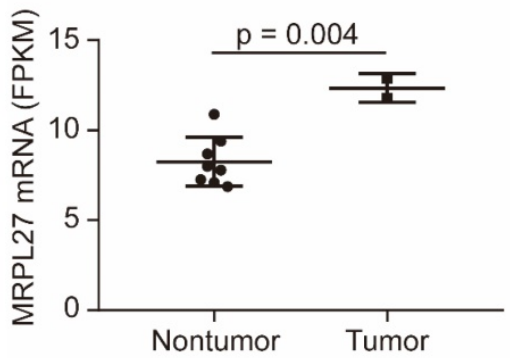

(E) MRPL27 protein staining in HPA

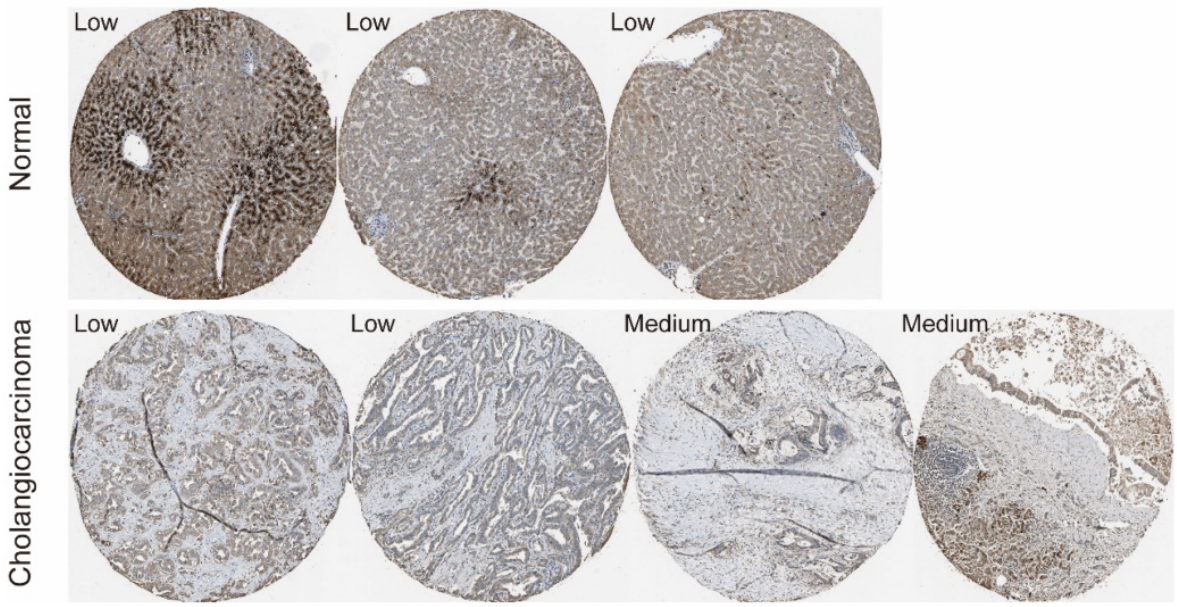

(B) Intrahepatic cholangiocarcinoma

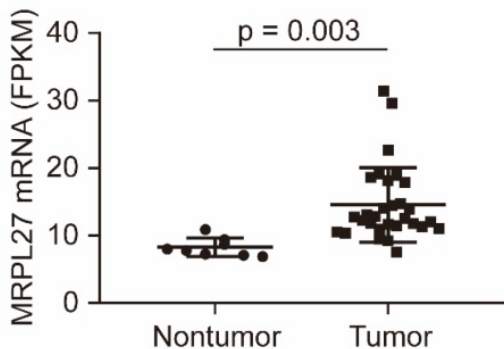

(D) Hilar/perihilar cholangiocarcinoma

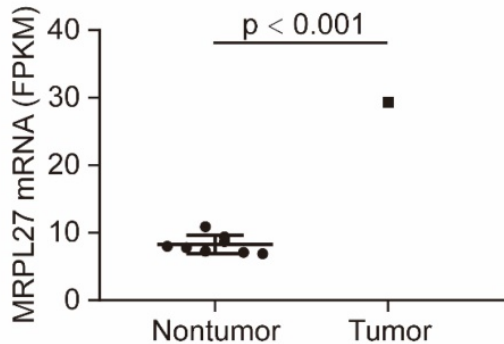

Figure 1. MRPL27 expression in cholangiocarcinoma patients.

Table 1. Baseline characteristics of cholangiocarcinoma patients in MRPL27 high and low expression groups

\begin{tabular}{llll}
\hline Variables & $\begin{array}{l}\text { MRPL27 high } \\
\text { group }(\mathrm{n}=18)\end{array}$ & $\begin{array}{l}\text { MRPL27 low } \\
\text { group }(\mathrm{n}=18)\end{array}$ & $\begin{array}{l}\mathrm{p} \\
\text { value }\end{array}$ \\
\hline Age, years, mean \pm SD & $64.1 \pm 2.5$ & $61.9 \pm 3.5$ & 0.62 \\
Male, $\mathrm{n}(\%)$ & $8(44.4)$ & $8(44.4)$ & 1.0 \\
Body mass index (BMI), n (\%) & & & 0.251 \\
BMI $<19.9$ & $2(11.1)$ & $0(0)$ & \\
$20 \leq \mathrm{BMI}<24.9$ & $5(27.8)$ & $3(16.7)$ & \\
$25 \leq \mathrm{BMI}<29.9$ & $8(44.4)$ & $8(44.4)$ & \\
BMI $\geq 30$ & $3(16.7)$ & $7(38.9)$ & 0.63 \\
White race, $\mathrm{n}(\%)$ & $16(88.9)$ & $15(83.3)$ & 0.735 \\
Family history of cancer, $\mathrm{n}(\%)$ & $10(55.6)$ & $11(61.1)$ & 0.735 \\
Risk factor of cancer, $\mathrm{n}(\%)$ & & & \\
No & $7(38.9)$ & $8(44.4)$ & \\
Yes & $11(61.1)$ & $10(55.6)$ & \\
Surgical therapy, $\mathrm{n}(\%)$ & & & \\
Other, Specify & $2(11.1)$ & $0(0)$ & \\
Segmentectomy, Single & $2(11.1)$ & $1(5.6)$ & \\
Pancreaticoduodenectomy, Whipple & $5(27.8)$ & $9(50.0)$ & \\
Resection & & & \\
\hline
\end{tabular}

\begin{tabular}{llll}
\hline Variables & $\begin{array}{l}\text { MRPL27 high } \\
\text { group }(\mathrm{n}=18)\end{array}$ & $\begin{array}{l}\text { MRPL27 low } \\
\text { group }(\mathrm{n}=18)\end{array}$ & $\begin{array}{l}\mathrm{p} \\
\text { value }\end{array}$ \\
\hline Segmentectomy, Multiple & $3(16.7)$ & $0(0)$ & \\
Extended Lobectomy & $3(16.7)$ & $4(22.2)$ & \\
Lobectomy & $3(16.7)$ & $4(22.2)$ & \\
Grade, $\mathrm{n}(\%)$ & & & 0.502 \\
I-II & $9(50.0)$ & $11(61.1)$ & \\
III-IV & $9(50.0)$ & $7(38.9)$ & \\
AJCC stage, $\mathrm{n}(\%)$ & & & 0.718 \\
I & $9(50.0)$ & $10(55.6)$ & \\
II & $4(22.2)$ & $5(27.8)$ & \\
III-IV & $5(27.8)$ & $3(16.7)$ & \\
Vascular invasion, $\mathrm{n}(\%)$ & $3(16.7)$ & $4(22.2)$ & 0.674 \\
Perineural invasion, $\mathrm{n}(\%)$ & $5(27.8)$ & $2(11.1)$ & 0.206 \\
Fibrosis (Ishak score), $\mathrm{n}(\%)$ & $6(33.3)$ & $5(27.8)$ & 0.717 \\
New tumor event after original treatment, & $10(55.6)$ & $7(38.9)$ & 0.317 \\
$\mathrm{n}$ (\%) & & & \\
Alpha-fetoprotein (AFP), mean \pm SD & $4.02 \pm 0.91$ & $3.44 \pm 0.53$ & 0.6 \\
Total bilirubin, mean \pm SD & $0.72 \pm 0.13$ & $0.64 \pm 0.07$ & 0.585 \\
Albumin, mean \pm SD & $4.13 \pm 0.12$ & $4.04 \pm 0.14$ & 0.652 \\
Creatinine, mean \pm SD & $0.85 \pm 0.06$ & $0.87 \pm 0.04$ & 0.732 \\
\hline
\end{tabular}




\section{Survival analysis of MRPL27 for cholangiocarcinoma patients}

In GEPIA online service, 36 cholangiocarcinoma patients were divided into MRPL27 high and low expression groups by median cutoff. As shown in Figure 2, cholangiocarcinoma patients with high MRPL27 in tumor tissues had significantly worse OS and DFS compared to those with low MRPL27 levels $(\mathrm{HR}=4.6, \mathrm{p}=0.004$ and $\mathrm{HR}=6.1, \mathrm{p}<0.001$, respectively, Figure $2 \mathrm{~A}$ and $2 \mathrm{~B}$ ). In validation set, Kaplan-Meier method revealed that high level of MRPL27 in tumor tissues contributed to poorer OS and DFS in cholangiocarcinoma patients $(p=0.022$ and $p=0.02$, respectively, Figure $2 \mathrm{C}$ and $2 \mathrm{D}$ ).

\section{Cox models for identification links between MRPL27 and survival in cholangiocarcinoma patients}

Variables including MRPL27, age, race, family history of cancer, history of hepatocarcinoma risk factors, surgical procedure, pathological histology grade, AJCC stage, vascular invasion, perineural

(A)

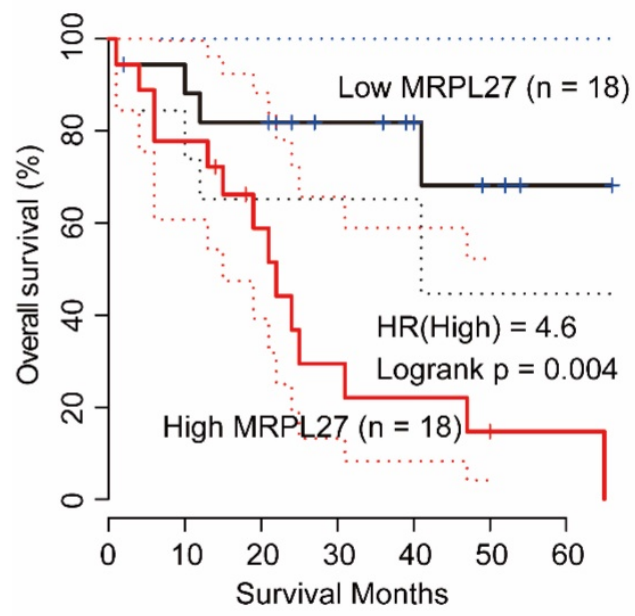

(C)

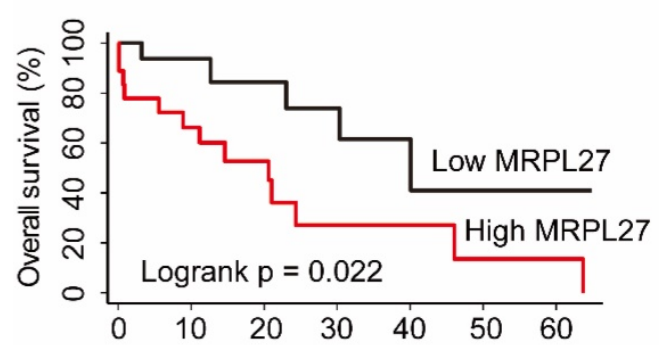

No. at risk survival months

$\begin{array}{rrrrrrr}18 & 11 & 9 & 6 & 3 & 1 & 1 \\ 18 & 11 & 7 & 3 & 2 & 1 & 1\end{array}$

invasion, ishak scores, new tumor event after original treatment, AFP, total bilirubin, albumin and creatinine were all included in the univariate Cox model. As shown in Table 2, MRPL27, surgical procedure, AJCC stage, vascular invasion, perineural invasion and new tumor event after original treatment might potential factors associated with OS in cholangiocarcinoma patients $(\mathrm{p}<0.10)$. After adjusted these indicators, MRPL27 and vascular invasion were risk factors for OS in cholangiocarcinoma patients in multivariate model $(\mathrm{HR}=4.99, \mathrm{p}=0.038$ and $\mathrm{HR}=11.13, \mathrm{p}=0.007$, respectively, Table 2 ).

As presented in Table 3, MRPL27, surgical procedure, vascular invasion and new tumor event after original treatment were potential indicators for DFS in cholangiocarcinoma patients $(\mathrm{p}<0.10)$. After adjusting surgical procedure, vascular invasion and new tumor event after original treatment, MRPL27 was identified as independent risk factor for DFS in cholangiocarcinoma patients $(\mathrm{HR}=5.72, \mathrm{p}=0.013$, Table 3)

(B)

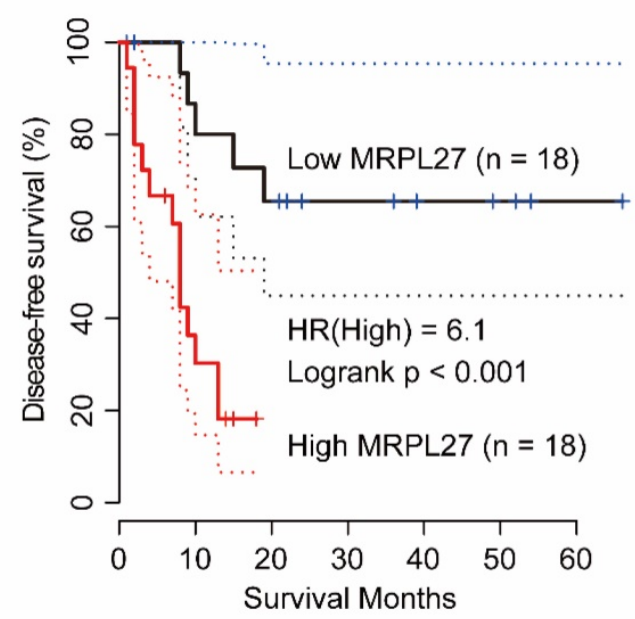

(D)

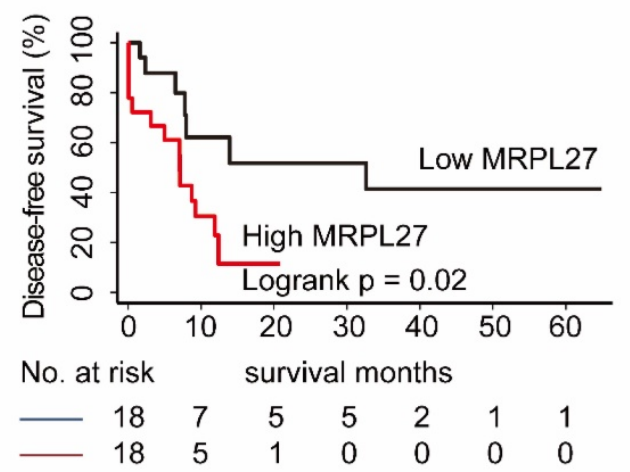

Figure 2. Overall survival and disease-free survival n MRPL27 high and low groups from cholangiocarcinoma patients in GEPIA database (A, B) and in LIHC profile n TCGA (C, D) 
Table 2. Parameters associated with OS in cholangiocarcinoma patients in TCGA database $\mathrm{A}^{\#}$

\begin{tabular}{|c|c|c|c|c|c|c|}
\hline \multirow[t]{2}{*}{ Variables } & \multicolumn{3}{|l|}{ Univariate } & \multicolumn{3}{|c|}{ Multivariate } \\
\hline & HR & $95 \% \mathrm{CI}$ & P value & HR & $95 \% \mathrm{CI}$ & P value \\
\hline \multicolumn{7}{|l|}{ MRPL27, median } \\
\hline Low & Reference & - & 1.0 & Reference & - & 1.0 \\
\hline High & 3.15 & $1.12-8.89$ & 0.03 & 4.99 & $1.09-22.82$ & 0.038 \\
\hline \multicolumn{7}{|l|}{ Surgical procedure } \\
\hline Other, Specify & Reference & - & 1.0 & Reference & - & 1.0 \\
\hline Segmentectomy, Single & 0.53 & $0.07-3.95$ & 0.537 & 0.10 & $0.01-1.30$ & 0.078 \\
\hline Pancreaticoduodenectomy, Whipple Resection & 0.10 & $0.02-0.59$ & 0.011 & 0.11 & $0.01-1.12$ & 0.063 \\
\hline Segmentectomy, Multiple & 0.17 & $0.01-1.92$ & 0.152 & 0.11 & $0-3.38$ & 0.209 \\
\hline Extended Lobectomy & 0.07 & $0.01-0.50$ & 0.009 & 0.08 & $0.01-1.16$ & 0.065 \\
\hline Lobectomy & 0.18 & $0.03-1.22$ & 0.078 & 0.24 & $0.02-2.30$ & 0.214 \\
\hline \multicolumn{7}{|l|}{ AJCC stage } \\
\hline I & Reference & - & 1.0 & Reference & - & 1.0 \\
\hline II & 2.98 & $0.91-9.76$ & 0.072 & 6.36 & $1.0-40.53$ & 0.05 \\
\hline III-IV & 1.78 & $0.56-5.64$ & 0.325 & 1.39 & $0.28-7.05$ & 0.689 \\
\hline \multicolumn{7}{|l|}{ Vascular invasion } \\
\hline No & Reference & - & 1.0 & Reference & - & 1.0 \\
\hline Yes & 3.15 & $1.03-9.66$ & 0.045 & 11.13 & $1.92-64.45$ & 0.007 \\
\hline \multicolumn{7}{|l|}{ Perineural invasion } \\
\hline No & Reference & - & 1.0 & Reference & - & 1.0 \\
\hline Yes & 3.56 & $0.97-13.07$ & 0.056 & 1.74 & $0.25-12.07$ & 0.576 \\
\hline \multicolumn{7}{|l|}{ New tumor event after original treatment } \\
\hline No & Reference & - & 1.0 & Reference & - & 1.0 \\
\hline Yes & 2.61 & $0.85-8.01$ & 0.094 & 1.70 & $0.26-11.11$ & 0.581 \\
\hline
\end{tabular}

Table 3. Parameters associated with DFS in cholangiocarcinoma patients in TCGA database $\#$

\begin{tabular}{|c|c|c|c|c|c|c|}
\hline \multirow[t]{2}{*}{ Variables } & \multicolumn{3}{|l|}{ Univariate } & \multicolumn{3}{|c|}{ Multivariate } \\
\hline & HR & $95 \% \mathrm{CI}$ & P value & HR & $95 \% \mathrm{CI}$ & P value \\
\hline \multicolumn{7}{|l|}{ MRPL27, median } \\
\hline Low & Reference & - & 1.0 & Reference & - & 1.0 \\
\hline High & 3.02 & $1.13-8.06$ & 0.027 & 5.72 & $1.45-22.60$ & 0.013 \\
\hline \multicolumn{7}{|l|}{ Surgical procedure } \\
\hline Other, Specify & Reference & - & 1.0 & Reference & - & 1.0 \\
\hline Segmentectomy, Single & 0.40 & $0.05-2.91$ & 0.365 & 0.34 & $0.04-2.57$ & 0.294 \\
\hline Pancreaticoduodenectomy, Whipple Resection & 0.26 & $0.05-1.28$ & 0.098 & 0.29 & $0.05-1.74$ & 0.175 \\
\hline Segmentectomy, Multiple & 0.11 & $0.01-1.35$ & 0.085 & 0.14 & $0.01-2.62$ & 0.189 \\
\hline Extended Lobectomy & 0.15 & $0.02-0.95$ & 0.044 & 0.21 & $0.03-1.49$ & 0.119 \\
\hline Lobectomy & 0.30 & $0.05-1.76$ & 0.183 & 0.38 & $0.06-2.60$ & 0.326 \\
\hline \multicolumn{7}{|l|}{ Vascular invasion } \\
\hline No & Reference & - & 1.0 & Reference & - & 1.0 \\
\hline Yes & 5.11 & $1.76-14.87$ & 0.003 & 13.14 & $2.97-58.10$ & 0.001 \\
\hline \multicolumn{7}{|l|}{ New tumor event after original treatment } \\
\hline No & Reference & - & 1.0 & Reference & - & 1.0 \\
\hline Yes & 5.20 & $1.71-15.74$ & 0.004 & 3.20 & $0.79-13.0$ & 0.103 \\
\hline
\end{tabular}

\section{Bioinformatic analysis of underlying functions of MRPL27}

To identify the underlying functions of MRPL27 in the development of carcinoma, bioinformatic analysis was performed. PPI analysis in STRING and STITCH indicated that MRPL2, MRPL4, MRPL11, MRPL13, MRPL17, MRPL19, MRPL21, MRPL22, MRPL24, MRPL29, MRPL33, MRPL36, MRPL47, MRPS15 and MRPS16 were interacted with MRPL27 (Figure 3A and 3B). Top 50 similar genes of MRPL27 in GEPIA database were summarized in Figure 3C.
Enrichment analysis of interacted and similar genes of MRPL27 showed that MRPL27 mainly involved in the processes of mitochondrial translation elongation, respiratory electron transport, ATP synthesis, and inner mitochondrial membrane organization, which were validated both in Metascape (Figure 4A) and GSEA (Figure 4B) databases. In addition, no mutations of MRPL27 were identified in cholangiocarcinoma patients via screening in cBioPortal for cancer genomics (Figure 4C). 
(A)

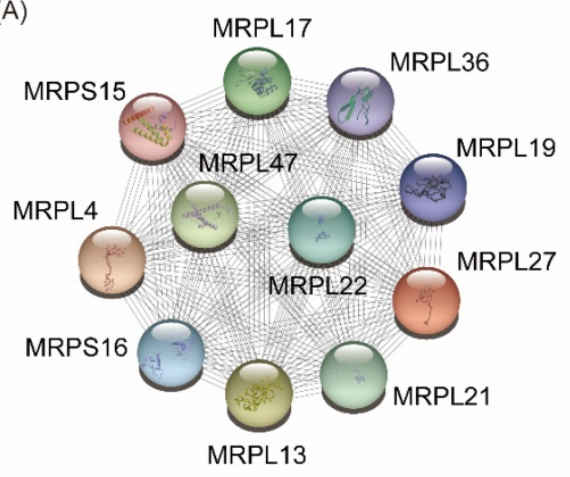

(C)

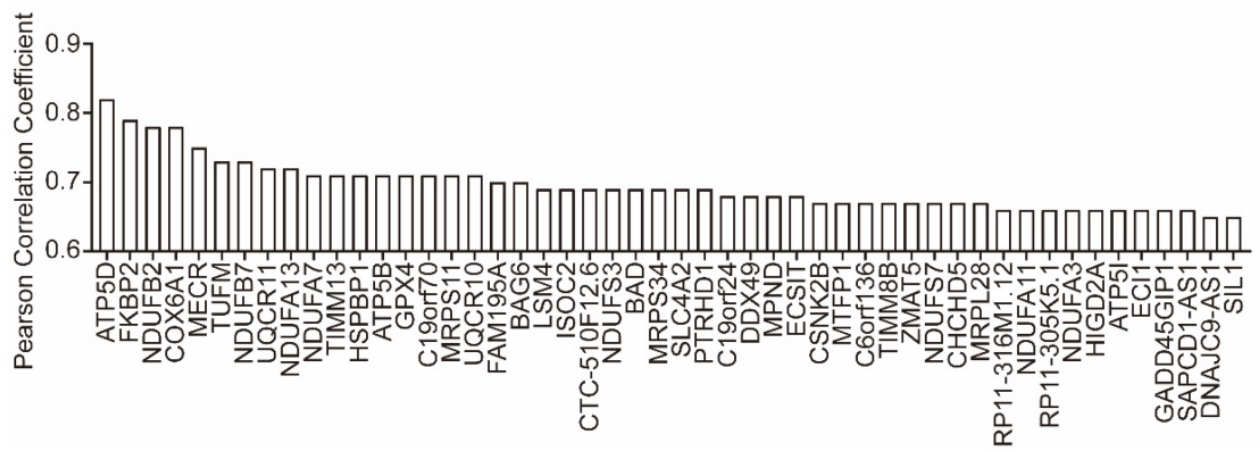

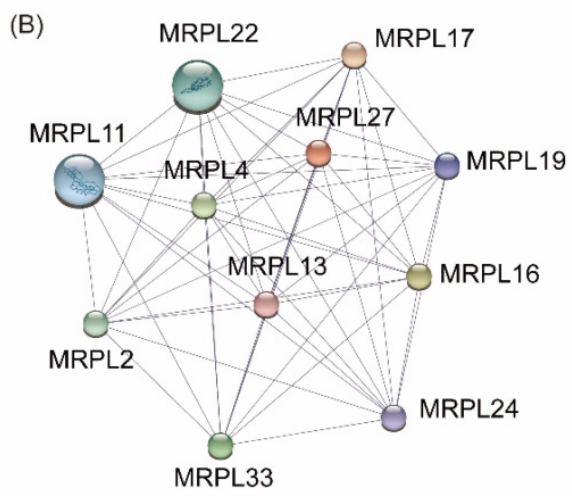

Figure 3. Protein-protein interaction in STRING (A) and STITCH (B), similar genes of MRPL27 in cholangiocarcinoma tumors in GEPIA (C).

(A)

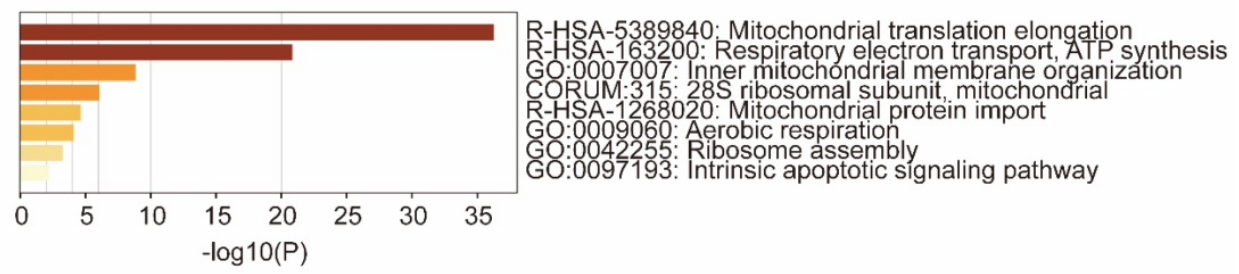

(B)

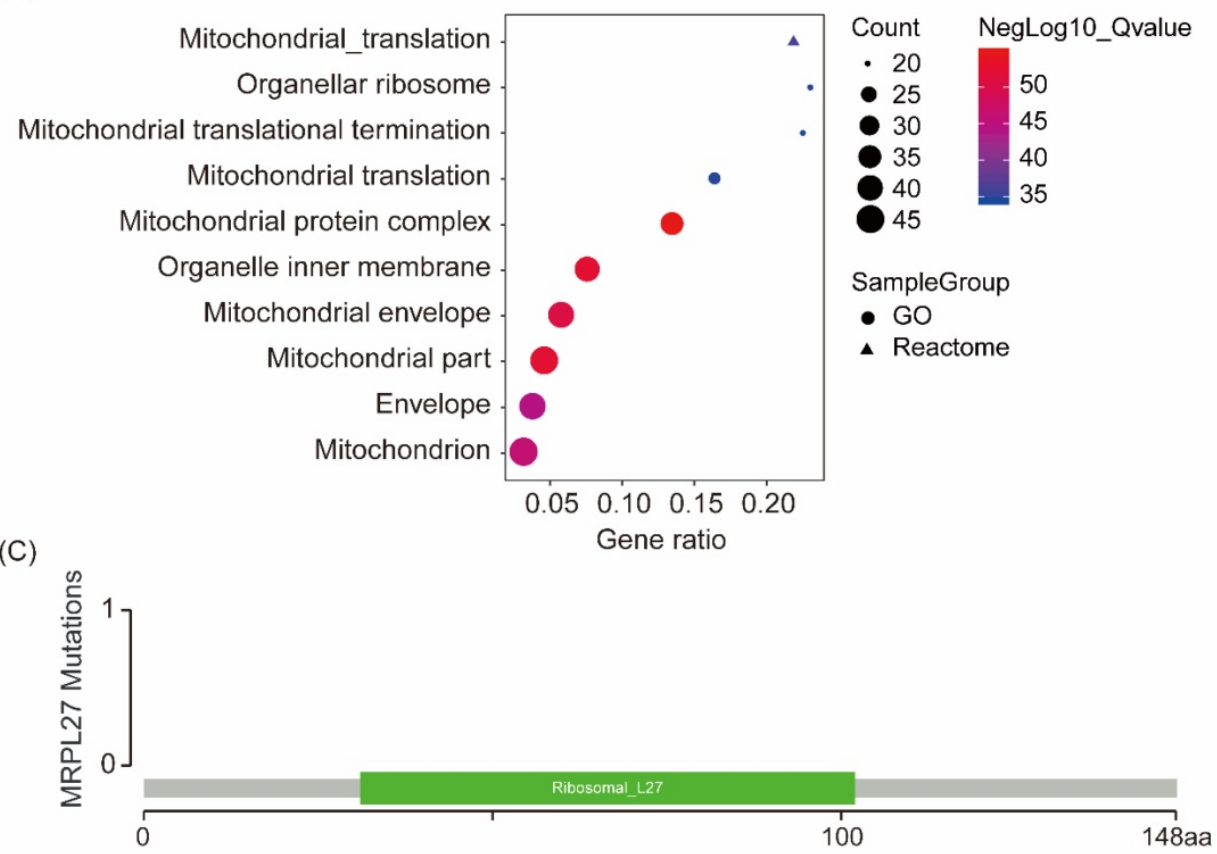

Figure 4. Enrichment of similar genes and interacted genes of MRPL27 in Metascape (A), GSEA (B), and MRPL27 mutation in chilangiocarcinoma from TCGA (C). 


\section{Discussion}

The therapeutic options of cholangiocarcinoma are very limited, resulting in that cholangiocarcinoma has a disappointing prognosis and is almost always irrecoverable owing to its refractory to most currently surgical procedures or medical interventions[18]. Except for tumor locations and pathological subtypes, genetic features also play vital roles in the diagnosis and prognosis in cholangiocarcinoma patients $[19,20]$. The huge genetic variations remain the main challenge of effective pharmacological therapy[18]. Currently, several molecular pathways including Notch, receptor tyrosine kinase pathways and PI3K-AKT-mTOR pathway, mutations including KRAS, IDH/IDH2, ROS1, FGFR and BAP1 and cytokines including interleukin-6 were identified dysregulated in cholangiocarcinoma[20].

Ribosome biogenesis serves as one important cellular process that is specially hyperactivated by neoplastic transformation and progression[21, 22]. Unfortunately, members in ribosome pathway were not well illustrated in cholangiocarcinoma. Even previous report uncovered that MRPL27 mRNA was downregulated in liver of the metabolic syndrome rats[23], our analysis indicated that MRPL27 was upregulated in cholangiocarcinoma and associated with unfavorable prognosis including OS and DFS. As a biomarker involved in ribosome production, dyskerin was positive in $56.7 \%$ cholangiocarcinoma patients and associated with p53 mutation and a higher proliferative index. Moreover, dyskerin was negatively correlated with DFS in cholangiocarcinoma patients[24]. Located in chromosome X, MRPL27 gene codes for 146 amino acids, YmL27 protein, and involves the large subunit of the mitochondrial ribosome[25-27]. In addition, MRPL27 is involved in mitochondrial translation as well as organelle biogenesis and maintenance[28], in which in line with our results from bioinformatic analysis. Gruschke $S$ et al revealed a complex network of interacting proteins including MBA1, MRPL3, MRPL13 as well as MRPL27 specific to mitochondrial ribosomes. And these proteins constitute ribosomal proteins exclusively found in mitochondria and promote oxidative phosphorylation in mitochondria[29]. Our bioinformatic analysis also indicated that MRPL27 contributed to ATP synthesis, which together with oxidative phosphorylation might involve in the tumor energy support system and contribute to cancer aggressiveness[30-32]. Additionally, no mutations of MRPL27 were screened in cholangiocarcinoma patients in our research. Taken together, we speculated that MRPL27 might be a potential candidate target for cancer therapy including cholangiocarcinoma.

Indeed, our study has some limitations. Firstly, this analysis included small samples of cholangiocarcinoma patients, leading to relatively low representative of this population. In our study, most of cholangiocarcinoma cases were intrahepatic cholangiocarcinoma, while the sample counts of distal cholangiocarcinoma and hilar/perihilar cholangiocarcinoma were low. Hence, the accuracy and reliability of these calculation results should be guaranteed in prospective studies with large samples. Secondly, we could not conduct experimental research for probing potential oncogenic mechanisms of MRPL27 in cholangiocarcinoma development. Thirdly, this analysis in a bioinformatic study based on TCGA dataset, there was no follow-up data for our own from available cholangiocarcinoma patients.

According to our preliminary findings, we cautiously concluded that upregulation of MRPL27 in tumor tissues predicted worse OS and DFS in cholangiocarcinoma patients. MRPL27 mainly involved in the processes of mitochondrial translation elongation, respiratory electron transport, ATP synthesis, and inner mitochondrial membrane organization.

\section{Acknowledgements}

This work was supported by National Natural Science Foundation of China (81803901) and Shanghai Anticancer Association EYAS project (SACA-CY1C16).

\section{Data availability}

Datasets of the current study are available from the corresponding author on reasonable request.

\section{Competing Interests}

The authors have declared that no competing interest exists.

\section{References}

1. Krasinskas AM. Cholangiocarcinoma. Surgical Pathology. 2018; 11: 403-29.

2. McGlynn KA, Tarone RE, El-Serag HB. A comparison of trends in the incidence of hepatocellular carcinoma and intrahepatic cholangiocarcinoma in the United States. Cancer Epidemiol Biomarkers Prev. 2006; 15: 1198-203.

3. Welzel TM, McGlynn KA, Hsing AW, et al. Impact of classification of hilar cholangiocarcinomas (Klatskin tumors) on the incidence of intra- and extrahepatic cholangiocarcinoma in the United States. J Natl Cancer Inst. 2006; 98: 873-5.

4. Alvaro D, Crocetti E, Ferretti S, et al. Descriptive epidemiology of cholangiocarcinoma in Italy. Dig Liver Dis. 2010; 42: 490-5.

5. Wood R, Brewster DH, Fraser LA, et al. Do increases in mortality from intrahepatic cholangiocarcinoma reflect a genuine increase in risk? Insights from cancer registry data in Scotland. Eur J Cancer. 2003; 39: 2087-92.

6. Blechacz B, Komuta M, Roskams $\mathrm{T}$, et al. Clinical diagnosis and staging of cholangiocarcinoma. Nat Rev Gastroenterol Hepatol. 2011; 8: 512-22.

7. Bertuccio P, Malvezzi M, Carioli G, et al. Global trends in mortality from intrahepatic and extrahepatic cholangiocarcinoma. J Hepatol. 2019; 71: 104-14.

8. Beal EW, Tumin D, Moris D, et al. Cohort contributions to trends in the incidence and mortality of intrahepatic cholangiocarcinoma. Hepatobiliary Surg Nutr. 2018; 7: 270-6.

9. Turi Z, Lacey M, Mistrik M, et al. Impaired ribosome biogenesis: mechanisms and relevance to cancer and aging. Aging (Albany NY). 2019; 11: 2512-40. 
10. Gao J, Aksoy BA, Dogrusoz U, et al. Integrative analysis of complex cancer genomics and clinical profiles using the cBioPortal. Sci Signal. 2013; 6: pl1.

11. Cerami E, Gao J, Dogrusoz U, et al. The cBio cancer genomics portal: an open platform for exploring multidimensional cancer genomics data. Cancer Discov. 2012; 2: 401-4.

12. Szklarczyk D, Gable AL, Lyon D, et al. STRING v11: protein-protein association networks with increased coverage, supporting functional discovery in genome-wide experimental datasets. Nucleic Acids Res. 2019; 47; D607-D13.

13. Szklarczyk D, Santos A, von Mering C, et al. STITCH 5: augmenting protein-chemical interaction networks with tissue and affinity data. Nucleic Acids Res. 2016; 44: D380-4.

14. Tang Z, Li C, Kang B, et al. GEPIA: a web server for cancer and normal gene expression profiling and interactive analyses. Nucleic Acids Res. 2017; 45: W98-W102.

15. Mootha VK, Lindgren CM, Eriksson KF, et al. PGC-1alpha-responsive genes involved in oxidative phosphorylation are coordinately downregulated in human diabetes. Nat Genet. 2003; 34: 267-73.

16. Subramanian A, Tamayo P, Mootha VK, et al. Gene set enrichment analysis: a knowledge-based approach for interpreting genome-wide expression profiles. Proc Natl Acad Sci U S A. 2005; 102: 15545-50.

17. Zhou Y, Zhou B, Pache L, et al. Metascape provides a biologist-oriented resource for the analysis of systems-level datasets. Nat Commun. 2019; 10: 1523.

18. Braconi C, Patel T. Cholangiocarcinoma: new insights into disease pathogenesis and biology. Infect Dis Clin North Am. 2010; 24: 871-84, vii.

19. Komuta M, Govaere O, Vandecaveye V, et al. Histological diversity in cholangiocellular carcinoma reflects the different cholangiocyte phenotypes. Hepatology. 2012; 55: 1876-88.

20. Rizvi S, Borad MJ, Patel T, et al. Cholangiocarcinoma: molecular pathways and therapeutic opportunities. Semin Liver Dis. 2014; 34: 456-64.

21. Derenzini M, Montanaro L, Trere D. Ribosome biogenesis and cancer. Acta Histochem. 2017; 119: 190-7.

22. Penzo M, Montanaro L, Trere D, et al. The Ribosome Biogenesis-Cancer Connection. Cells. 2019; 8 .

23. Zheng N, Wei D, Dai B, et al. Mitochondrial Genome Encoded Proteins Expression Disorder, the Possible Mechanism of the Heart Disease in Metabolic Syndrome. Cell Physiol Biochem. 2017; 43: 959-68.

24. Vasuri F, Rocchi L, Degiovanni A, et al. Dyskerin expression in human fetal, adult and neoplastic intrahepatic bile ducts: correlations with cholangiocarcinoma aggressiveness. Histopathology. 2015; 66: 244-51.

25. Graack HR, Grohmann L, Kitakawa M. The nuclear coded mitoribosomal proteins YmL27 and YmL31 are both essential for mitochondrial function in yeast. Biochimie. 1991; 73: 837-44.

26. Holmstrom K, Brandt T, Kallesoe T. The sequence of a 32,420 bp segment located on the right arm of chromosome II from Saccharomyces cerevisiae. Yeast. 1994; 10 Suppl A: S47-62.

27. Daniel J. Direct in vivo access to potential gene targets of the RPD3 histone deactylase using fitness-based interferential genetics. Yeast. 2007; 24: 575-87.

28. Bogatikov E, Lindblad I, Punga T, et al. miR-1933-3p is upregulated in skeletal muscles of MuSK+ EAMG mice and affects Impa1 and Mrpl27. Neurosci Res. 2020; 151: 46-52.

29. Gruschke S, Grone K, Heublein M, et al. Proteins at the polypeptide tunnel exit of the yeast mitochondrial ribosome. J Biol Chem. 2010; 285: 19022-8.

30. Ashton TM, McKenna WG, Kunz-Schughart LA, et al. Oxidative Phosphorylation as an Emerging Target in Cancer Therapy. Clin Cancer Res. 2018; 24: 2482-90.

31. Sun $\mathrm{H}$, Zhang $\mathrm{C}$, Cao $\mathrm{S}$, et al. Fenton reactions drive nucleotide and ATP syntheses in cancer. J Mol Cell Biol. 2018; 10: 448-59.

32. Maddocks OD, Labuschagne CF, Adams PD, et al. Serine Metabolism Supports the Methionine Cycle and DNA/RNA Methylation through De Novo ATP Synthesis in Cancer Cells. Mol Cell. 2016; 61: 210-21. 